Journal of Difference Equations and Applications,

Vol. 00, No. 00, Month 200x, 1-6

\title{
Global Stability for Mixed Monotone Systems
}

\author{
H.L. Smith* \\ Department of Mathematics and Statistics, Arizona State University, Tempe, AZ 85287 \\ (v3.1 released August 2006)
} We show that the embedding method described in $[4,8]$ leads immediately to the global stability results in [7]. It also allows extension
of some results on global stability for higher order difference equations due to Gerry Ladas and collaborators. Further, we provide a new
result which suggests that embedding into monotone systems may not be necessary for global stability results.

Keywords: mixed monotone system, monotone dynamical system, global stability

This paper is dedicated to Gerry Ladas on the occasion of his 70th Birthday

\section{Introduction}

The idea of embedding a dynamical system, whose generator has both increasing and decreasing monotonicity properties (positive and negative feedback), into a larger symmetric monotone dynamical system and exploiting the convergence properties of the latter is very old. For a discussion of history of the method, see $[4,8]$. The method is repeatedly rediscovered and its implications are often underestimated. In this paper, we review the main results of the embedding method following [8] and then we show how it leads immediately to an improved version of a nice result on global stability due to Kulenović and Merino [7] for componentwise monotone maps that leave invariant a hypercube in Euclidean space. We then ask whether embedding a system into a larger monotone system is really necessary to obtain global stability results. On the face of it, it seems unnatural to pass to a larger dimensional dynamical system in order to gain information on the dynamics of a smaller one. We show that for the class of mixed-monotone systems, one can obtain global stability results directly without the need of embedding.

As noted in [8], the embedding method leads to a nice generalization of some results of Kulenović, Ladas and Sizer [5], also contained in the monograph of Kulenović and Ladas [6], on higher order difference equations with componentwise monotonicity.

\section{Review of the Embedding Method}

Let $X$ be an ordered metric space with closed order relation $\leq$. The closedness of the order relation means that if $x_{n} \leq y_{n}$ and $x_{n} \rightarrow x, y_{n} \rightarrow y$, then $x \leq y$. If $x \leq y$, define the order interval $[x, y]:=\{z \in X: x \leq$ $z \leq y\}$. Let $F: X \rightarrow X$ be continuous. Our focus is the discrete-time dynamical system defined by

$$
x^{\prime}=F(x)
$$

where $x^{\prime}$ denotes the successor to $x$.

*Corresponding author. Email: halsmith@asu.edu

Journal of Difference Equations and Applications

ISSN: 1023-6198 print/ISSN 1563-5120 online (c) 200x Taylor \& Francis

http://www.tandf.co.uk/journals

DOI: 10.1080/1023619YYXxxxxxxx 
We say $F$ is mixed-monotone if there exists a continuous map $f: X \times X \rightarrow X$ satisfying:

(1) $F(x)=f(x, x), x \in X$.

(2) $\forall y \in X, x_{1}, x_{2} \in X, x_{1} \leq x_{2} \Rightarrow f\left(x_{1}, y\right) \leq f\left(x_{2}, y\right)$.

(3) $\forall x \in X, y_{1} \leq y_{2} \Rightarrow f\left(x, y_{2}\right) \leq f\left(x, y_{1}\right)$.

In short, $f$ is nondecreasing in its first variable and nonincreasing in its second. Roughly, $F$ is a map that combines both positive and negative feedback. We write $F^{n}(x)$ for the $n$-fold composition of $F$ acting on $x$. The omega limit set of a subset $A \subset X$ is denoted by $\omega_{F}(A)$ and that of a single point $x \in X$, is denoted by $\omega_{F}(x)$.

As shown in [4], (1) can be embedded in the symmetric discrete-time dynamical system

$$
\begin{aligned}
& x^{\prime}=f(x, y) \\
& y^{\prime}=f(y, x)
\end{aligned}
$$

on $X \times X$. We use the notation $z=(x, y)$ and define

$$
G(z)=G(x, y)=(f(x, y), f(y, x))
$$

$G$ is called the symmetric map. Obviously, the diagonal

$$
D=\{(x, x): x \in X\}
$$

is positively invariant under (2) and $G(x, x)=(F(x), F(x))$. The symmetry of $G$ can be expressed by defining the reflection operator $R(x, y)=(y, x)$ and observing that $G \circ R=R \circ G$.

The "southeast" ordering on $X^{2}:=X \times X$ is the closed partial order relation defined by

$$
(x, y) \leq_{C}(\bar{x}, \bar{y}) \Longleftrightarrow x \leq \bar{x} \text { and } \bar{y} \leq y .
$$

It's name derives from the fact that the bigger point lies southeast of the smaller one. Note that $R: X^{2} \rightarrow$ $X^{2}$ is order reversing.

Although the map $F$ need not be monotone, the symmetric map $G$ is monotone.

Lemma 2.1: $G$ is monotone with respect to $\leq_{C}$ on $X \times X$ in the sense that

$$
z \leq_{C} \bar{z} \Longrightarrow G(z) \leq_{C} G(\bar{z}) .
$$

Moreover, the "above-diagonal set" $\{(x, y) \in X \times X: x \leq y\}$ is positively invariant under $G$.

The following result, proved in [8] (see also [2]), is a sharpened version of Theorem 7 in [4].

Theorem 2.2: Suppose that:

$$
\exists x_{0}, y_{0}, x_{0} \leq y_{0}, \text { satisfying } f\left(x_{0}, y_{0}\right) \geq x_{0}, f\left(y_{0}, x_{0}\right) \leq y_{0} .
$$

Then $F\left(\left[x_{0}, y_{0}\right]\right) \subset\left[x_{0}, y_{0}\right]$ and for $z_{0}=\left(x_{0}, y_{0}\right)$ and $n \geq 1$, we have:

$$
z_{0} \leq_{C} G^{n}\left(z_{0}\right) \leq_{C} G^{n+1}\left(z_{0}\right) \leq_{C} R z_{0} .
$$

Assume, in addition, that the monotone orbit

$$
\left\{G^{n}\left(x_{0}, y_{0}\right)\right\}_{n \geq 1} \text { converges in } X \text {. }
$$


Then:

(i) there exist $x_{*}, y_{*} \in\left[x_{0}, y_{0}\right]$ with $x_{*} \leq y_{*}$ satisfying

$$
G^{n}\left(x_{0}, y_{0}\right) \longrightarrow\left(x_{*}, y_{*}\right)=G\left(x_{*}, y_{*}\right)
$$

implying that $f\left(x_{*}, y_{*}\right)=x_{*}, f\left(y_{*}, x_{*}\right)=y_{*}$.

(ii) If $x \in\left[x_{0}, y_{0}\right]$ and $\left\{F^{n}(x)\right\}_{n \geq 1}$ has compact closure in $X$, then

$$
\omega_{F}(x) \subset\left[x_{*}, y_{*}\right]
$$

(iii) If $F\left(\left[x_{0}, y_{0}\right]\right)$ has compact closure in $X$, then $\omega_{F}\left(\left[x_{0}, y_{0}\right]\right) \neq \emptyset$, and

$$
\omega_{F}\left(\left[x_{0}, y_{0}\right]\right) \subset\left[x_{*}, y_{*}\right] .
$$

(iv) If

$$
a, b \in\left[x_{0}, y_{0}\right], a \leq b, f(a, b)=a, b=f(b, a) \Rightarrow a=b
$$

holds then $x_{*}=y_{*}$ and $F\left(x_{*}\right)=x_{*}$. In this case, if $x \in\left[x_{0}, y_{0}\right]$ and $\left\{F^{n}(x)\right\}_{n \geq 1}$ has compact closure in $X$, then $\omega_{F}(x)=\left\{x_{*}\right\}$.

As noted in [8], the hypothesis (4) may be satisfied under a variety of hypotheses on either the space $X$ or the map $f$. If $X$ is a finite dimensional ordered Banach space or if $X=L^{p}(\Omega)$ is a Lebesgue space then order bounded sequences converge. It holds if $f$ has compactness properties. See [8].

The proof of Theorem 2.2 exploits the fact that (3) is equivalent to:

$$
\left(x_{0}, y_{0}\right) \leq_{C} G\left(x_{0}, y_{0}\right)
$$

and so the monotone symmetric map $G$ has a monotone increasing (relative to $\left.\leq_{C}\right)$ orbit $\left(x_{n}, y_{n}\right)=$ $G^{n}\left(x_{0}, y_{0}\right)$ which must remain in the "above-diagonal" set $\{(x, y) \in X \times X: x \leq y\}$ by Lemma 2.1. By monotonicity of $G$ and the fact that $G=(F, F)$ on the diagonal, one concludes that for $x \in\left[x_{0}, y_{0}\right]$ we have

$$
x_{n} \leq F^{n}(x) \leq y_{n}
$$

where $x_{n} \nearrow x_{*}$ and $y_{n} \searrow y_{*}$.

Applications of Theorem 2.2 can be found in [8]. See also those in [7].

\section{A result of Kulenović and Merino}

We show that the main result of Kulenovic and Merino [7] follows from Theorem 2.2.

Let $m, M \in \mathrm{R}^{\mathrm{k}}$ satisfy $m \leq M$ and let $F:[m, M] \rightarrow[m, M]$ be a continuous map. Kulenović and Merino call $F$ coordinate-wise monotone (cw-monotone) if $F_{i}$ is monotone in $x_{j}$ on $\left[m_{j}, M_{j}\right]$ for $1 \leq i, j \leq k$. For each $i, K=\{1,2, \cdots, k\}$ can be partitioned into two disjoint subsets as follows:

$$
I_{i}=\left\{j \in K: F_{i} \text { is nondecreasing or constant in } x_{j}\right\}
$$

and

$$
D_{i}=\left\{j \in K: F_{i} \text { is nonincreasing and nonconstant in } x_{j}\right\}
$$


Given vectors $x, y \in[m, M]$ denote by $\left(x_{I_{i}}, y_{D_{i}}\right)$ the vector $z \in r m R^{k}$ with $z_{l}=x_{l}$ for $l \in I_{i}$ and $z_{l}=y_{l}$ for $l \in D_{i}$. Observe that $z \in[m, M]$. Define $f:[m, M] \times[m, M] \rightarrow \mathrm{R}^{\mathrm{k}}$ by

$$
f_{i}(x, y)=F\left(x_{I_{i}}, y_{D_{i}}\right)
$$

Kulenović and Merino give an alternate but equivalent definition of $f$ as follows. Define

$$
\sigma_{i j}=\left\{\begin{array}{cc}
1 & \text { if } F_{i} \text { is nondecreasing or constant in } x_{j} \\
-1 & \text { if } F_{i} \text { is nonincreasing and nonconstant in } x_{j}
\end{array}\right\}
$$

Then for $1 \leq i \leq k$,

$$
f_{i}(x, y)=F_{i}\left(\frac{1+\sigma_{i 1}}{2} x_{1}+\frac{1-\sigma_{i 1}}{2} y_{1}, \frac{1+\sigma_{i 2}}{2} x_{2}+\frac{1-\sigma_{i 2}}{2} y_{2}, \cdots, \frac{1+\sigma_{i k}}{2} x_{k}+\frac{1-\sigma_{i k}}{2} y_{k}\right)
$$

Observe that

(a) $f([m, M] \times[m, M]) \subset[m, M]$.

(b) $F(x)=f(x, x), x \in[m, M]$.

(c) $f$ is nondecreasing in $x$ and nonincreasing in $y$.

The following result extends Theorem 3 of Kulenović and Merino [7].

Theorem 3.1: Let $F:[m, M] \rightarrow[m, M]$ be a continuous cw-monotone map. Assume that

$$
a, b \in[m, M], a \leq b, f(a, b)=a, b=f(b, a) \Rightarrow a=b
$$

holds. Then there exists $x_{*} \in[m, M]$ such that $\omega_{F}(x)=x_{*}$ for all $x \in[m, M]$.

Proof: Since $f:[m, M] \times[m, M] \rightarrow[m, M]$, it follows that $f(m, M) \geq m$ and $f(M, m) \leq M$. Hence hypothesis (3) of Theorem 2.2 holds with $x_{0}=m$ and $y_{0}=M$. Indeed, all hypotheses of that theorem hold so the result follows from part (iv).

Theorem 3.1 extends Theorem 3 in [7] because the additional restriction $a \leq b$ appears in our premise (8) but not in the premise of II. of Theorem 3 of [7].

\section{To Embed or not to Embed}

One might wonder whether it is really necessary to embed (1) into the symmetric map (2) in order to obtain significant results. We begin by showing that a seemingly more powerful hypothesis than (8) is actually equivalent to it.

Proposition 4.1: Suppose that hypotheses (3) and (4) of Theorem 2.2 hold. Then hypothesis (8) holds if and only if

$$
a, b \in\left[x_{0}, y_{0}\right], a \leq b, f(a, b) \leq a, b \leq f(b, a) \Rightarrow a=b
$$

holds.

Proof: Suppose that (8) holds and that there exists $a, b \in\left[x_{0}, y_{0}\right]$ such that $a \leq b, f(a, b) \leq a, b \leq f(b, a)$. In terms of the symmetric map $G$, this means that $G(a, b) \leq_{C}(a, b)$. Then $\left(x_{0}, y_{0}\right) \leq_{C}(a, b)$ and so by monotonicity of $G$

$$
\left(x_{0}, y_{0}\right) \leq_{C} G\left(x_{0}, y_{0}\right) \leq_{C} G(a, b) \leq_{C}(a, b) .
$$


It follows that $G\left(x_{*}, y_{*}\right)=\left(x_{*}, y_{*}\right)=\lim _{n} G^{n}\left(x_{0}, y_{0}\right) \leq_{C}(a, b)$. So $f\left(x_{*}, y_{*}\right)=x_{*}, f\left(y_{*}, x_{*}\right)=y_{*}$ and $x_{*} \leq y_{*}$. (8) implies that $x_{*}=y_{*}$. The inequality $\left(x_{*}, y_{*}\right) \leq_{C}(a, b)$ implies that $b \leq y_{*}=x_{*} \leq a$ which, together with $a \leq b$ implies $a=b$.

Now suppose that $X$ is a nonempty subset of an ordered metric space $Z$. We say that the continuous map $F: X \rightarrow X$ is weakly mixed-monotone if there exists a (not necessarily continuous) map $f: X \times X \rightarrow Z$ (note the range space is $Z$ !) satisfying:

(1) $F(x)=f(x, x), x \in X$.

(2) $\forall y \in X, x_{1}, x_{2} \in X, x_{1} \leq x_{2} \Rightarrow f\left(x_{1}, y\right) \leq f\left(x_{2}, y\right)$.

(3) $\forall x \in X, y_{1} \leq y_{2} \Rightarrow f\left(x, y_{2}\right) \leq f\left(x, y_{1}\right)$.

Theorem 4.2: $\quad$ Suppose that $F$ is weakly mixed monotone and:

$$
a, b \in X, a \leq b, f(a, b) \leq a, b \leq f(b, a) \Rightarrow a=b .
$$

If $X$ contains the infimum and supremum of any pair of fixed points of $F$, then $F$ has at most one fixed point in $X$.

If $x \in X$ has the property that $\overline{\left\{F^{t}(x)\right\}_{t=0}^{t=\infty}}$ is compact and contained in $X$ and such that inf $\omega_{F}(x)$ and $\sup \omega_{F}(x)$ exist in $Z$ and belong to $X$, then $\omega_{F}(x)$ is a fixed point of $F$.

Proof: First, observe that (10) implies uniqueness of fixed points for $F$ in $X$. If $f(a, a)=a$ and $f(b, b)=b$ for $a, b \in X$, set $A=\inf \{a, b\}$ and $B=\sup \{a, b\}$. Then $A, B \in X$ by hypothesis and $A \leq a, b \leq B$. By the weak mixed monotone condition, $f(A, B) \leq f(a, a)=a$ and $f(A, B) \leq f(b, b)=b$ so $f(A, B) \leq A$. Similarly, $f(B, A) \geq B$. Therefore, $A=B$ and $a=b$ by (10).

Suppose that $x \in X$ has the property that $\overline{\left\{F^{t}(x)\right\}_{t=0}^{t=\infty}}$ is compact and contained in $X$ and such that $c=\inf \omega_{F}(x)$ and $d=\sup \omega_{F}(x)$ exist in $Z$ and belong to $X$. Therefore $\omega_{F}(x)$ is nonempty and invariant: $F\left(\omega_{F}(x)\right)=\omega_{F}(x)$. If $y \in \omega_{F}(x)$, there exists $z \in \omega_{F}(x)$ such that $F(z)=y$. By the properties of $f$ and the fact that $c \leq z \leq d$, we conclude that $f(c, d) \leq y=F(z)=f(z, z) \leq f(d, c)$. Therefore, $f(c, d) \leq \omega(x) \leq f(d, c)$ which implies that $f(c, d) \leq c$ and $f(d, c) \geq d$. (10) implies that $c=d$. Therefore, $\omega_{F}(x)$ is a singleton, necessarily a fixed point.

In the special case that $X=\left[x_{0}, y_{0}\right] \subset \mathrm{R}^{\mathrm{k}}$ with any orthant ordering or $X=\left[x_{0}, y_{0}\right] \subset C\left(A, \mathrm{R}^{\mathrm{k}}\right)$ where $A$ is a compact space and $C\left(A, \mathrm{R}^{\mathrm{k}}\right)$ is ordered in the natural way from some orthant ordering on $\mathrm{R}^{\mathrm{k}}$, and if orbits of $F$ are precompact, then the second part of Theorem 4.2 extends Theorem2.2 (iv). Indeed, compact subsets of $C\left(A, \mathrm{R}^{\mathrm{k}}\right)$ have infima and suprema.

Observe from the proof of Theorem 4.2 that the map $f$ is used in a very limited way compared to its use in Theorem2.2. Unlike its use in the proof of Theorem2.2 where it defines the map $G$, in the proof of Theorem 4.2 there is no need to iterate $f$, nor is its continuity required. These facts allow weakening the hypotheses on $f$ for Theorem 4.2 .

\section{References}

[1] C. Cosner, Comparison Principles for systems that embed in cooperative systems, with applications to diffusive Lotka-Volterra Models, Dynam. Continuous, Discrete, and Impulsive Systems, 3 (1997), 283-303.

[2] G. Enciso, E. Sontag and H.L. Smith, Non-monotone systems decomposable into monotone systems with negative feedback, Journal of Differential Equations, Volume 224, Issue 1, 1 May 2006, 205-227.

[3] J.-L. Gouzé, A criterion of global convergence to equilibrium for differential systems with an application to Lotka-Volterra systems, Rapport de Recherche 894, 1988 INRIA.

[4] J.-L. Gouzé and K.P. Hadeler, Monotone Flows and order intervals, Nonlinear World 1, 1994, 23-34.

[5] M. Kulenović and G. Ladas, Dynamics of second order rational difference equations, Chapman \& Hall/CRC, Boca Raton Fl. 2002. 
[6] M. Kulenović, G. Ladas, W.Sizer, On the recursive sequence $x_{n+1}=\left(\alpha x_{n}+\beta x_{n-1}\right) /\left(\gamma x_{n}+\delta x_{n-1}\right)$, Math. Sci. Res. Hot-Line 2(5) 1998,1-16.

[7] M. Kulenović and O. Merino, A global attractivity result for maps with invariant boxes, Discrete and Cont. Dynamical Systems, ser.B, 6 (2006) 97-110.

[8] H.L. Smith, The discrete dynamics of monotonically decomposable maps, J. Math. Biol. 53, 2006, 747-758. 